\title{
Incidence and distribution of UroSEEK gene panel in a multi- institutional cohort of bladder urothelial carcinoma
}

\author{
Marie-Lisa Eich ${ }^{1} \cdot$ Maria Del Carmen Rodriguez Pena ${ }^{1} \cdot$ Simeon U. Springer ${ }^{2,3} \cdot$ Diana Taheri $^{4,5} \cdot$ Aline C. Tregnago $^{4}$. \\ Daniela C. Salles ${ }^{4}$ - Stephania Martins Bezerra ${ }^{6,7} \cdot$ Isabela W. Cunha ${ }^{6}{ }^{6} \cdot$ Kazutoshi Fujita $^{8} \cdot$ Dilek Ertoy $^{9}$. \\ Trinity J. Bivalacqua ${ }^{10} \cdot$ Cristian Tomasetti $^{11,12} \cdot$ Nickolas Papadopoulos $^{2,3} \cdot$ Ken W. Kinzler $^{2,3} \cdot$ Bert Vogelstein $^{2,3}$. \\ George J. Netto (iD) ${ }^{1}$
}

Received: 29 January 2019 / Revised: 22 March 2019 / Accepted: 23 March 2019 / Published online: 25 April 2019

(c) United States \& Canadian Academy of Pathology 2019

\begin{abstract}
Noninvasive approaches for early detection of bladder cancer are actively being investigated. We recently developed a urinebased molecular assay for the detection and surveillance of bladder neoplasms (UroSEEK). UroSEEK is designed to detect alterations in 11 genes that include most common genetic alterations in bladder cancer. In this study, we analyzed 527 cases, including 373 noninvasive and 154 invasive urothelial carcinomas of bladder from transurethral resections or cystectomies performed at four institutions (1991-2016). Two different mutational analysis assays of a representative tumor area were performed: first, a singleplex PCR assay for evaluation of the TERT promoter region (TERTSeqS) and second, a multiplex PCR assay using primers designed to amplify regions of interest of 10 (FGFR3, PIK3CA, TP53, HRAS, KRAS, ERBB2, $C D K N 2 A, M E T, M L L$, and $V H L$ ) genes (UroSeqS). Overall, 92\% of all bladder tumors were positive for at least one genetic alteration in the UroSEEK panel. We found TERT promoter mutations in $77 \%$ of low-grade noninvasive papillary carcinomas, with a relatively lower incidence of $65 \%$ in high-grade noninvasive papillary carcinomas and carcinomas in situ; $p=0.017$. Seventy-two percent of pT1 and $63 \%$ of muscle-invasive bladder tumors harbored TERT promoter mutations with g. 1295228C $>\mathrm{T}$ alteration being the most common in all groups. FGFR3 and PIK3CA mutations were more frequent in lowgrade noninvasive papillary carcinomas compared with high-grade noninvasive papillary carcinomas and carcinomas in situ $(p<0.0001)$, while the opposite was true for TP53 $(p<0.0001)$. Significantly higher rates of TP53 and CDKN2A mutation rates $(p=0.005$ and 0.035 , respectively) were encountered in muscle-invasive bladder tumors compared with those of pT1 stage. The overwhelming majority of all investigated tumors showed at least one mutation among UroSEEK assay genes, confirming the comprehensive coverage of the panel and supporting its potential utility as a noninvasive urinebased assay.
\end{abstract}

Supplementary information The online version of this article (https:// doi.org/10.1038/s41379-019-0276-y) contains supplementary material, which is available to authorized users.

George J. Netto

gnetto@uabmc.edu

1 Department of Pathology, The University of Alabama at Birmingham, Birmingham, AL, USA

2 Howard Hughes Medical Institute, Ludwig Cancer for Cancer Genetics and Therapeutics, Baltimore, MD, USA

3 Sidney Kimmel Comprehensive Cancer Center, Baltimore, MD, USA

4 Department of Pathology, Johns Hopkins University, Baltimore, MD, USA

5 Department of Pathology, Isfahan Kidney Diseases Research Center, Isfahan University of Medical Sciences, Isfahan, Iran
6 Department of Pathology, AC Camargo Cancer Center, Sao Paulo, Brazil

7 Department of Pathology, Rede D’OR-São Luiz, Sao Paulo, Brazil

8 Department of Urology, Osaka University, Osaka, Japan

9 Department of Pathology, Hacettepe University, Ankara, Turkey

10 Department of Urology, Johns Hopkins University, Baltimore, MD, USA

11 Department of Biostatistics, Johns Hopkins Bloomberg School of Public Health, Baltimore, MD, USA

12 Division of Biostatistics and Bioinformatics, Department of Oncology, Sidney Kimmel Cancer Center, Baltimore, MD, USA 


\section{Introduction}

Bladder cancer is the fourth most common cancer in men and the most common malignancy of the urinary tract in both male and female. In 2019, 80,470 new cases are being estimated in the Unites States, leading to 17,670 deaths [1].

Invasive urothelial carcinoma evolves through two distinct pathways: low- and high-grade noninvasive papillary urothelial carcinoma and "flat" carcinoma in situ [2]. Due to high recurrence rates and likelihood of progression to muscle-invasive bladder cancer, periodical follow-up with cystoscopy and cytology for patients diagnosed with noninvasive tumors is required [3-6]. These procedures entail an estimated $\$ 3$ billion burden to the health care system every year [7]. Alternative approaches for surveillance are therefore needed.

Early detection of bladder cancer remains a challenge in clinical practice. Hematuria, with or without lower urinary tract symptoms, is the most common presenting symptom. While asymptomatic microscopic hematuria is prevalent (up to $31 \%$ ) [8,9], only a small fraction of patients will ultimately be diagnosed with bladder cancer (3-5\%) [10]. Therefore, risk stratification with a noninvasive method to avoid unneeded cystoscopy is of great utility. Desquamated urothelial cells in urine have long been used as a valuable source for noninvasive detection of bladder cancer. Beside cystoscopy, urine cytology remains the gold standard for bladder cancer detection. However, its overall low sensitivity (11-76\%), especially in low-grade tumors, tampers its utility. Several FDA- approved urine-based noninvasive assays are currently available (e.g., UroVysion, Immuno$\mathrm{Cyt} / \mathrm{uCyt}^{\mathrm{TM}}, \mathrm{NMP} 22^{\circ}$, and BTA ${ }^{\circ}$ ) for both early detection and surveillance of bladder cancer. Sensitivity and specificity for these assays range from 56 to $78 \%$ and 74 to $88 \%$, respectively [11-18]. We recently developed a noninvasive bladder cancer assay with promising performance characteristics in both early detection and surveillance setting. When combined with cytology, a sensitivity of $95 \%$ and a specificity of $93 \%$ were reached in the early detection cohort [19]. The assay, termed "UroSEEK", consists of three components (TERTSeqS, UroSeqS, and FastSeqS). It covers molecular alterations that are frequently encountered in bladder cancer, in addition to aneuploidy (FastSeqS). The alterations include TERT promoter mutations (TERTSeqS) that occur in up to $80 \%$ of bladder cancers $[20,21]$ and 10 additional genes: FGFR3, PIK3CA, HRAS, KRAS, TP53, CDKN2A, ERBB2, MLL, $M E T$, and $V H L$ (UroSeqS) [22-24].

Evidently, a noninvasive mutation-based approach can only be effective if its genes are closely matched to the tumors it aims to detect. Therefore, in this study, we sought to identify the distribution of the UroSEEK gene panel in archival tumor tissues from a multi-institutional international cohort of bladder urothelial carcinoma. The relationship with tumor grade and stage was also assessed.

\section{Materials and methods}

\section{Patient samples and clinical data}

The study was approved by the Institutional Board Review of participating institutions. The required material transfer agreements were obtained. The 527 formalin-fixed, paraffin-embedded bladder urothelial carcinoma specimens were collected between 1991 and 2016 from four international academic institutions (Johns Hopkins Hospital, Baltimore, MD, United States; A.C. Camargo Cancer Center, Sao Paulo, Brazil; Osaka University Hospital, Osaka, Japan; and Hacettepe University Hospital, Ankara, Turkey). The mutational findings of the UroSEEK gene panel in a subset (102 tumors) were described in our previously reported study, describing the noninvasive multigene assay (UroSEEK) [19]. All histologic sections from transurethral resection of bladder tumor and cystectomy specimens were reviewed by a genitourinary pathologist, to confirm the diagnosis and select a representative tumor area. The corresponding formalin-fixed, paraffin-embedded blocks were cored for DNA purification as previously described [20]. Clinicopathologic data were obtained from electronic medical records. Only cases with a minimum follow-up time of 3 months were included in outcome analysis. Disease recurrence was defined as the development of histologically documented tumor occurrence. Progression was defined as the occurrence of histologically documented upgrade or upstage of disease.

\section{Mutation analysis}

Mutation and data analysis were performed as previously described [19, 20, 25, 26]. In brief, purified DNA was submitted for SafeSeqS analysis, a sequencing errorreduction technique capable of discriminating mutations from artifactual sequencing variants introduced during the sequencing process [27, 28]. Two different mutational analysis assays were performed: first, a singleplex PCR assay for evaluation of TERT promoter region (TERTSeqS) and a second multiplex PCR assay [20] using primers designed to amplify regions of interest of 10 (FGFR3, PIK3CA, TP53, HRAS, KRAS, ERBB2, CDKN2A, MET, $M L L$, and $V H L$ ) genes (UroSeqS). Primers are listed in Supplementary Table S1 [19].

To evaluate the statistical significance of observed mutations, DNA from white blood cells of 188 unrelated healthy individuals was also assessed. A variant was scored as a mutation only if the mutant allele frequency was much 
higher than that observed in normal white blood cells. As previously described [19], the classification of a sample's DNA status was based on two complementary criteria applied to each mutation: 1) the difference between the average mutant allele frequency in the sample of interest and the corresponding maximum mutant allele frequency observed for that same mutation in a set of controls; and 2) the Stouffer's Z-score obtained by comparing the mutant allele frequency in the sample of interest to a distribution of normal controls.

\section{Statistical analysis}

Statistical analysis was performed using $\mathrm{R}$ version 3.5.1 (07-02-2018) from the R Foundation for Statistical Computing (Vienna, Austria). For hypothesis testing, statistical significance was established at $p<0.05$ for two tails of distribution. The relationship between mutation status and pathological and outcome variables was analyzed by a Chisquare test with Yates' continuity correction.

\section{Results}

\section{Clinicopathologic features}

Five hundred and twenty-seven tumors from 484 patients were included in the study. One-hundred and thirteen patients were female and 371 were male. The median age was 68 years (range 28-96). Three hundred and twenty-nine patients were Caucasian, 68 were Asian, and 46 were African-American with the race in the remaining 41 patients being undetermined or from other category. The tumor included 188 low-grade noninvasive papillary carcinomas, 129 high-grade noninvasive papillary carcinomas, 56 carcinomas in situ, and 154 high-grade invasive urothelial carcinomas, including 111 pT1 and $43 \geq$ pT2 (muscleinvasive) tumors.

\section{Mutation analysis}

The frequency of mutations of all genes of the UroSEEK gene panel across histopathological categories is summarized in Fig. 1. The mutation variants of analyzed genes are listed in Supplementary Table S2. Overall, 93\% of noninvasive and $92 \%$ of invasive tumors were positive for at least one mutation in genes included in UroSEEK assay.

TERT promoter mutations were identified in $70 \%$ of all cases, with the most common alteration being g. $1295228 \mathrm{C}>\mathrm{T}$, followed by g. $1295250 \mathrm{C}>\mathrm{T}$. A previously unreported variant (g.1295223G $>\mathrm{T}$, in one case) was detected. A higher incidence of TERT promoter mutation was identified in low-grade noninvasive papillary carcinomas compared with high-grade noninvasive papillary carcinomas and carcinomas in situ $(77 \%$ vs. $65 \%$; $p=0.017$; see Table 1). Although a higher frequency of TERT promoter mutations occurred in pT1 cases $(72 \%)$ compared with muscle-invasive bladder cancer (63\%), the difference was not statistically significant.

Among the 10 genes included in the UroSeqS assay, FGFR3 and PIK3CA mutations occurred significantly more often in low-grade noninvasive papillary carcinoma tumors compared with high-grade noninvasive papillary carcinomas and carcinomas in situ $(p<0.0001)$, while the reverse was true for TP53 $(p<0.0001$; see Table 1$)$. In invasive bladder cancer, CDKN2A and TP53 mutations were more commonly observed in muscle-invasive bladder cancer compared with pT1 tumors ( $P=0.035$ and 0.005 , respectively; see Table 2$)$. Regarding mutation variants, p.S249C was the most frequent FGFR3 mutation (67\%), while p.E545K and p.H66P were the most common PIK3CA and CDKN2A mutations (49 and $79 \%$, respectively; see Supplementary Table S2).

Two hundred and nineteen tumors demonstrated the cooccurrence of mutations in both assay components, with $59 \%$ of tumors harboring TERT promoter mutation, also showing a mutation in at least one UroSeqS gene $(p=0.001)$. Finally, occasional cases demonstrated multiple variants of TERT promoter (13 tumors) and TP53 (14 tumors) mutations, and one case displayed two FGFR3 variant mutations.

\section{Mutational analysis in patients with sequential tumors}

Tissues from sequential tumors were available for mutational analysis in 36 patients. As illustrated in Fig. 2, TERT promoter mutations were absent in all samples in 7 of the 36 patients. In the remaining 29 patients, the same TERT promoter mutation was persistently present across tumors in 22 and variably found in 7 patients. Only 2 of 15 patients with observed FGFR3 mutations had the same mutation present across tumors.

\section{Association of UroSEEK assay with outcome}

The distribution of the three hundred and three cases that met the minimum follow-up requirement for outcome analysis was as follows: 124 low-grade noninvasive papillary carcinomas, 78 high-grade noninvasive papillary carcinomas, 24 carcinomas in situ, 58 pT1, and 19 muscle-invasive bladder tumors.

The association of tumor UroSEEK, TERTSeqS, and UroSeqS findings with recurrence and progression are summarized in Table 3. As shown, 94\% of tumors with subsequent recurrence had mutation in at least one of the 11 genes included in UroSEEK. A comparable rate of $95 \%$ was found in tumors without recurrence $(p=0.784)$. Only 21 


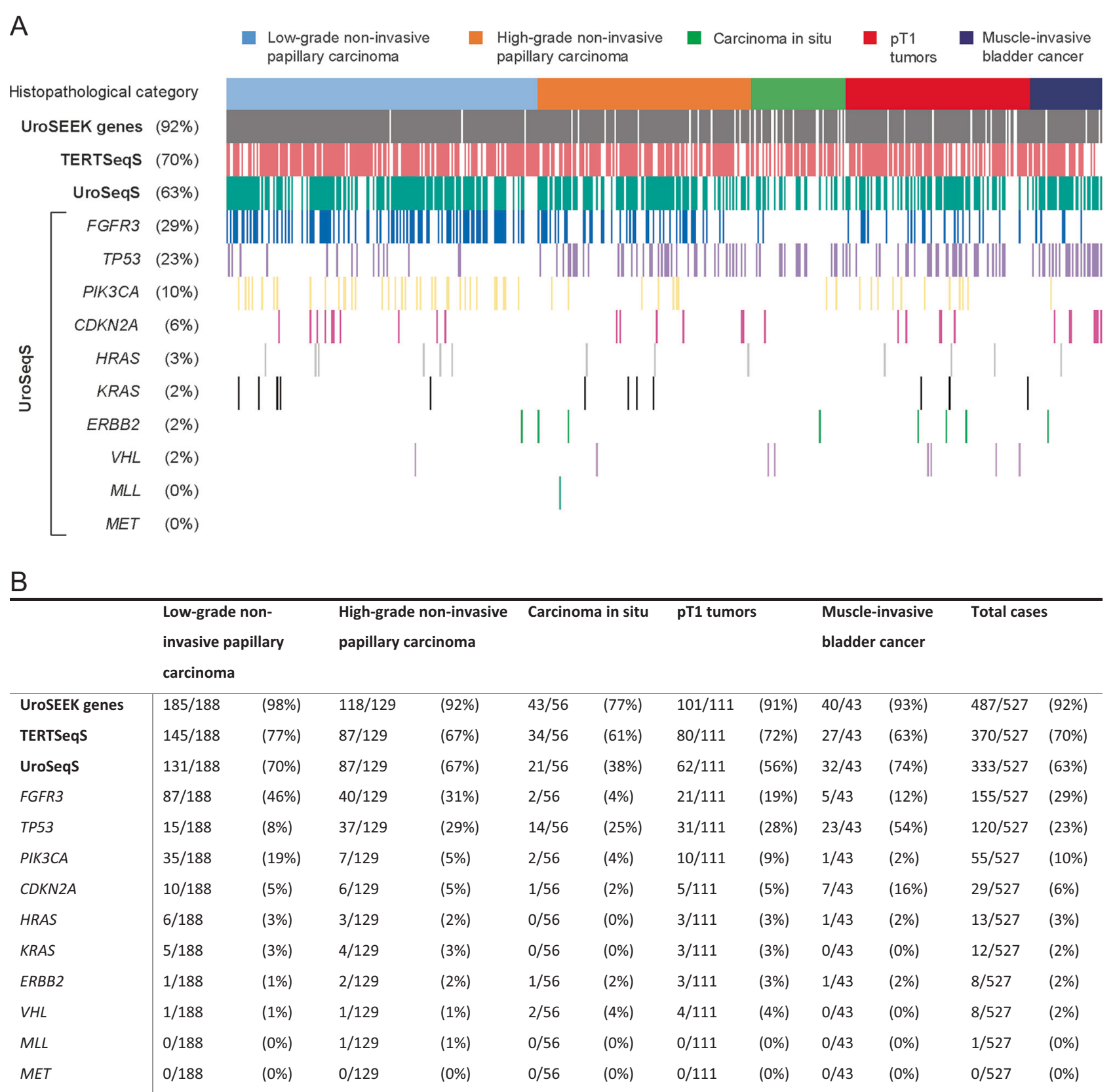

Fig. 1 Distribution of mutations in the UroSEEK gene panel and its TERTSeqS and UroSeqS components across 527 bladder carcinomas. a Oncoplot graphical representation of data. Each column represents

patients developed progression during follow-up. Tumors with progression were less likely to harbor a mutation in one or more of the UroSEEK assay genes ( $81 \%$ vs. $96 \%$; $p=0.016$ ).

\section{Discussion}

In our current multicenter study, we investigated the comprehensive coverage of the UroSEEK gene assay in archival one tumor. The top line colored boxes indicate the histopathological category. Rows indicate affected gene(s) and their rate of mutations. Detailed listing of the absolute numbers of mutations is shown in b

bladder cancer tissues. The TERTSeqS component detected mutations in the TERT promoter region in $70 \%$ of all tumors. Mutations in the UroSeqS gene panel were found in $63 \%$ of all cases. Combining these two components led to a capture rate for alteration of the UroSEEK assay in $92 \%$ of all tumors. More specifically, in the subset of low-grade noninvasive papillary carcinoma cases, we found at least one gene alteration in $98 \%$ of the cases, with $77 \%$ being positive for TERTSeqS and 70\% positive for UroSeqS. This is especially important, given the low sensitivity of routine 
Table 1 Distribution of mutations of the UroSEEK gene panel in noninvasive bladder cancer

\begin{tabular}{lllc}
\hline & $\begin{array}{l}\text { Low-grade } \\
\text { noninvasive } \\
\text { papillary } \\
\text { carcinoma }\end{array}$ & $\begin{array}{l}\text { High-grade } \\
\text { noninvasive } \\
\text { papillary } \\
\text { carcinoma }+\end{array}$ & $p$-value \\
carcinoma in situ & \\
\hline UroSEEK genes & $185 / 188(98 \%)$ & $161 / 185(87 \%)$ & $<\mathbf{0 . 0 0 0 1}$ \\
TERTSeqS & $145 / 188(77 \%)$ & $121 / 185(65 \%)$ & $\mathbf{0 . 0 1 7}$ \\
UroSeqS & $131 / 188(70 \%)$ & $108 / 185(58 \%)$ & $\mathbf{0 . 0 3 0}$ \\
FGFR3 & $87 / 188(46 \%)$ & $42 / 185(23 \%)$ & $<\mathbf{0 . 0 0 0 1}$ \\
TP53 & $15 / 188(8 \%)$ & $51 / 185(28 \%)$ & $<\mathbf{0 . 0 0 0 1}$ \\
PIK3CA & $35 / 188(19 \%)$ & $9 / 185(5 \%)$ & $<\mathbf{0 . 0 0 0 1}$ \\
CDKN2A & $10 / 188(5 \%)$ & $7 / 185(4 \%)$ & 0.644 \\
HRAS & $6 / 188(3 \%)$ & $3 / 185(2 \%)$ & 0.515 \\
KRAS & $5 / 188(3 \%)$ & $4 / 185(2 \%)$ & 1.0 \\
ERBB2 & $1 / 188(1 \%)$ & $3 / 185(2 \%)$ & 0.604 \\
VHL & $1 / 188(1 \%)$ & $3 / 185(2 \%)$ & 0.604 \\
$M L L$ & $0 / 188(0 \%)$ & $1 / 185(1 \%)$ & 0.994 \\
MET & $0 / 188(0 \%)$ & $0 / 185(0.0 \%)$ & $\cdot$ \\
\hline A & &
\end{tabular}

Assay categories (UroSEEK and its components) and significant $p$-value are listed in bold

Table 2 Distribution of mutations of the UroSEEK gene panel in invasive bladder cancer

\begin{tabular}{llll}
\hline & pT1 tumors & $\begin{array}{l}\text { Muscle- } \\
\text { invasive } \\
\text { bladder cancer }\end{array}$ & $p$-value \\
\hline UroSEEK genes & $101 / 111(91 \%)$ & $40 / 43(93 \%)$ & 0.933 \\
TERTSeqS & $80 / 111(72 \%)$ & $27 / 43(63 \%)$ & 0.354 \\
UroSeqS & $62 / 111(56 \%)$ & $32 / 43(74 \%)$ & 0.053 \\
FGFR3 & $21 / 111(19 \%)$ & $5 / 43(12 \%)$ & 0.399 \\
TP53 & $31 / 111(28 \%)$ & $23 / 43(54 \%)$ & $\mathbf{0 . 0 0 5}$ \\
PIK3CA & $10 / 111(9 \%)$ & $1 / 43(2 \%)$ & 0.273 \\
CDKN2A & $5 / 111(5 \%)$ & $7 / 43(16 \%)$ & $\mathbf{0 . 0 3 5}$ \\
HRAS & $3 / 111(3 \%)$ & $1 / 43(2 \%)$ & 1.0 \\
KRAS & $3 / 111(3 \%)$ & $0 / 43(0 \%)$ & 0.661 \\
ERBB2 & $3 / 111(3 \%)$ & $1 / 43(2 \%)$ & 1.0 \\
VHL & $4 / 111(4 \%)$ & $0 / 43(0 \%)$ & 0.486 \\
MLL & $0 / 111(0 \%)$ & $0 / 43(0 \%)$ & $\cdot$ \\
MET & $0 / 111(0 \%)$ & $0 / 43(0 \%)$ & $\cdot$ \\
\hline
\end{tabular}

Assay categories (UroSEEK and its components) and significant $p$-value are listed in bold

cytology for the diagnosis of low-grade noninvasive tumors. As evidenced in our recent study, urine cytology was negative in all 49 low-grade noninvasive papillary carcinoma cases, while urine UroSEEK was positive in $2 / 3$ of these cases [19].

The current observation of $54 \%$ of TP53 mutation in muscle-invasive bladder cancer is in line with findings of recent studies, such as The Cancer Genome Atlas [23] and Kim et al. [29], where TP53 was altered in 48 and $57 \%$ of cases, respectively. We observed a lower frequency for $P I K 3 C A, E R B B 2$, and $M L L$ in muscle-invasive bladder cancer compared with these two studies.

Our study represents one of the largest assessments of a multigene assay in low-grade noninvasive papillary carcinoma tumors to date, where 188 cases were analyzed. In this group, FGFR 3 mutations were the most frequent (46\%) among the 10 genes included in UroSeqS, followed by PIK3CA mutation in $19 \%$. These rates are lower than those obtained by Hurst et al. [30] in their study of whole-exome and targeted sequencing of 82 Ta tumors (79\% and $54 \%$ for FGFR3 and PIK3CA mutations, respectively). This could be due to our hotspot-focused mutational analysis approach, given that Hurst et al. have identified many non-hotspot mutations in their analysis.

TERT promoter mutations are one of the most frequent alterations in bladder cancer and its variants [25, 26, 3133]. In this analysis, we found TERT promoter mutations in $70 \%$ of all cases, with the highest rate observed in lowgrade noninvasive papillary carcinomas (77\%). The herefound distribution rates of TERT promoter mutations in noninvasive lesions are in line with our originally reported rates in conventional urothelial carcinoma in Kinde et al., where the low-grade noninvasive papillary carcinoma group had the highest rate of TERT promoter mutations of $86 \%$ [20]. In contrast, Pietzak et al. found TERT promoter mutations to be more frequent in their high-grade noninvasive papillary carcinoma cases compared with lowgrade noninvasive papillary carcinomas $(88 \%$ and $61 \%$, respectively) [34].

Our analysis of sequential tumors in 36 patients unveiled an identical TERT promoter mutation across tumors in 22 of the 29 patients harboring TERT promoter mutations. In surveillance setting, UroSEEK is envisioned to be used for follow-up in patients, whose index tumor is positive for at least one alteration in the panel. This consistency of TERT promoter alteration across tumors, if proven in a larger cohort of sequential tumors, suggests that assessment of the index tumor might be sufficient and could obviate the need for repeat sequential tumor testing. The same approach would not apply to cases where the index tumor is only positive for one of the ten genes in UroSeqS, where we found inconsistency in mutation detection across tumors from the same patient. This might in part be due to tumor molecular heterogeneity that was not captured by our adopted technique.

Given the nature of the cohort in the original study describing the noninvasive multigene assay (UroSEEK) [19], analysis of association with outcome could only be performed in a subset of patients. This is due to the fact that in a proportion of cases, tumors that are contemporaneous 


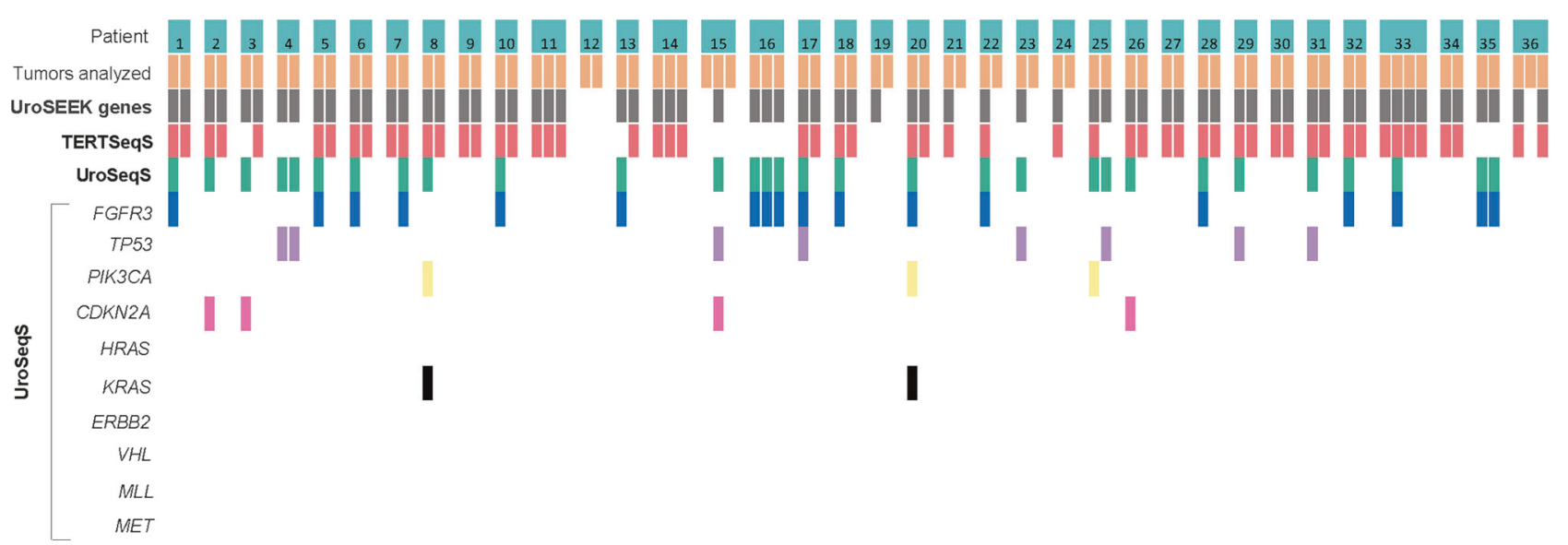

Fig. 2 Oncoplot representation of mutations in the UroSEEK gene panel and its TERTSeqS and UroSeqS components in 36 patients with sequential tumors. Each broad column represents one patient. The

individual tumor per patient (orange box) and their mutated gene(s) are indicated in each row
Table 3 Association of the UroSEEK gene panel with outcome

\begin{tabular}{lllllll}
\hline & Recurrence & & $P$-value & Progression & $p$-value \\
\hline & Yes & No & & Yes & No & \\
& $N=132$ & $N=171$ & & $N=21$ & $N=282$ & \\
Mutation in UroSEEK genes & $124 / 132$ & $163 / 171$ & 0.784 & $17 / 21$ & $270 / 282$ & $\mathbf{0 . 0 1 6}$ \\
& $(94 \%)$ & $(95 \%)$ & & $(81 \%)$ & $(96 \%)$ & \\
Mutation in TERTSeqS & $101 / 132$ & $115 / 171$ & 0.101 & $12 / 21$ & $204 / 282$ & 0.217 \\
& $(77 \%)$ & $(67 \%)$ & & $(57 \%)$ & $(72 \%)$ & \\
Mutation in UroSeqS & $87 / 132$ & $120 / 171$ & 0.505 & $12 / 21$ & $195 / 282$ & 0.369 \\
& $(66 \%)$ & $(70 \%)$ & & $(57 \%)$ & $(69 \%)$ & \\
\hline
\end{tabular}

Assay categories (UroSEEK and its components) and significant $p$-value are listed in bold with analyzed urine samples were selected. The strengths of the study include the large number of cases in the multiinstitutional cohort (527 tumors) and the advantageous error-proof nature of the SafeSeqS technique.

In conclusion, the overwhelming majority of all investigated tumors showed at least one mutation among genes included in the recently reported UroSEEK assay, across tumor grade and stage. This confirms the comprehensive coverage of the UroSEEK panel and supports its potential utility as a noninvasive urine-based assay. Our findings are especially reassuring in the subset of low-grade noninvasive papillary carcinoma, where routine cytology lacks sensitivity.

Acknowledgements We are grateful for the generous support provided by the Johns Hopkins Greenberg Bladder Cancer Institute, Henry and Marsha Laufer, the Virginia and DK Ludwig Fund for Cancer Research, the Commonwealth Foundation, the John Templeton Foundation, and the Conrad R Hilton Foundation. All sequencing was performed at the Sol Goldman Sequencing Facility at Johns Hopkins. This work was also supported by grants from the NIH (Grants CA77598, CA 06973, GM 07309, and ES019564).

\section{Compliance with ethical standards}

Conflict of interest NP, KWK, and BV: founders of Personal Genome Diagnostics and PapGene and advisors to Sysmex-Inostics. KWK and BV also advise Eisai. BV is also an advisor to Camden Partners. These companies and others have licensed technologies from Johns Hopkins that are related to the work described in this paper. These licenses are associated with equity or royalty payments to NP, KWK, GJN, and $\mathrm{BV}$. Additional patent applications on the work described in this paper may be filed by Johns Hopkins University. The terms of these arrangements are managed by the university in accordance with its conflict of interest policies. The remaining authors declare that they have no conflict of interest.

Publisher's note: Springer Nature remains neutral with regard to jurisdictional claims in published maps and institutional affiliations.

\section{References}

1. Siegel RL, Miller KD, Jemal A. Cancer statistics, 2019. CA Cancer J Clin. 2019;69:7-34.

2. Netto GJ. Clinical applications of recent molecular advances in urologic malignancies: no longer chasing a "mirage"? Adv Anat Pathol. 2013;20:175-203. 
3. Chamie K, Litwin MS, Bassett JC, Daskivich TJ, Lai J, Hanley JM, et al. Recurrence of high-risk bladder cancer: a populationbased analysis. Cancer. 2013;119:3219-27.

4. Millan-Rodriguez F, Chechile-Toniolo G, Salvador-Bayarri J, Palou J, Algaba F, Vicente-Rodriguez J. Primary superficial bladder cancer risk groups according to progression, mortality and recurrence. J Urol. 2000;164:680-4.

5. Chang SS, Boorjian SA, Chou R, Clark PE, Daneshmand S, Konety BR, et al. Diagnosis and treatment of non-muscle invasive bladder cancer: AUA/SUO Guideline. J Urol. 2016;196:1021-9.

6. Babjuk M, Bohle A, Burger M, Capoun O, Cohen D, Comperat $\mathrm{EM}$, et al. EAU guidelines on non-muscle-invasive urothelial carcinoma of the bladder: update 2016. Eur Urol. 2017;71:447-61.

7. Netto GJ, Epstein JI. Theranostic and prognostic biomarkers: genomic applications in urological malignancies. Pathology. 2010;42:384-94.

8. Wood PW. Urothelial Tumors of the Bladder. In: Wein AJ, Kavoussi LR, Novick AC, Partin AW, Peters CA, editors. Campbell-Walsh Urology. 10th edn. Philadelphia, PA Elsevier Saunders, 2012. p.2309-34.

9. Davis R, Jones JS, Barocas DA, Castle EP, Lang EK, Leveillee RJ, et al. Diagnosis, evaluation and follow-up of asymptomatic microhematuria $(\mathrm{AMH})$ in adults: AUA guideline. $\mathrm{J}$ Urol. 2012;188:2473-81.

10. Davis R, Jones JS, Barocas DA et al. Diagnosis, Evaluation and Follow-up of Asymptomatic Microhematuria (AMH) in Adults, 2016, [cited 10/10/2018]. https://www.auanet.org/guidelines/a symptomatic-microhematuria-(amh)-guideline

11. Smith ZL, Guzzo TJ. Urinary markers for bladder cancer. F1000Prime Rep. 2013;5:21.

12. O'Sullivan P, Sharples K, Dalphin M, Davidson P, Gilling P, Cambridge L, et al. A multigene urine test for the detection and stratification of bladder cancer in patients presenting with hematuria. J Urol. 2012;188:741-7.

13. Wang Z, Que H, Suo C, Han Z, Tao J, Huang Z, et al. Evaluation of the NMP22 BladderChek test for detecting bladder cancer: a systematic review and meta-analysis. Oncotarget. 2017;8:100648-56.

14. He H, Han C, Hao L, Zang G. ImmunoCyt test compared to cytology in the diagnosis of bladder cancer: a meta-analysis. Oncol Lett. 2016;12:83-8.

15. Lavery HJ, Zaharieva B, McFaddin A, Heerema N, Pohar KS. A prospective comparison of UroVysion FISH and urine cytology in bladder cancer detection. BMC Cancer. 2017;17:247.

16. Lokeshwar VB, Habuchi T, Grossman HB, Murphy WM, Hautmann SH, Hemstreet GP, et al. Bladder tumor markers beyond cytology: International Consensus Panel on bladder tumor markers. Urology. 2005;66:35-63. https://doi.org/10.1016/j.urology. 2005.08.064

17. Guo A, Wang X, Gao L, Shi J, Sun C, Wan Z. Bladder tumour antigen (BTA stat) test compared to the urine cytology in the diagnosis of bladder cancer: a meta-analysis. Can Urol Assoc J. 2014;8:E347-52.

18. Chou R, Gore JL, Buckley D, Fu R, Gustafson K, Griffin JC, et al. Urinary biomarkers for diagnosis of bladder cancer: a systematic review and meta-analysis. Ann Intern Med. 2015;163:922-31.
19. Springer SU, Chen CH, Rodriguez Pena MDC, Li L, Douville C, Wang Y, et al. Non-invasive detection of urothelial cancer through the analysis of driver gene mutations and aneuploidy. Elife. 2018;7:pii: e43237.

20. Kinde I, Munari E, Faraj SF, Hruban RH, Schoenberg M, Bivalacqua $\mathrm{T}$, et al. TERT promoter mutations occur early in urothelial neoplasia and are biomarkers of early disease and disease recurrence in urine. Cancer Res. 2013;73:7162-7.

21. Killela PJ, Reitman ZJ, Jiao Y, Bettegowda C, Agrawal N, Diaz LA, et al. TERT promoter mutations occur frequently in gliomas and a subset of tumors derived from cells with low rates of selfrenewal. Proc Natl Acad Sci USA. 2013;110:6021-6.

22. Hedegaard J, Lamy P, Nordentoft I, Algaba F, Hoyer S, Ulhoi BP, et al. Comprehensive transcriptional analysis of early-stage urothelial carcinoma. Cancer Cell. 2016;30:27-42.

23. Robertson AG, Kim J, Al-Ahmadie H, Bellmunt J, Guo G, Cherniack AD, et al. Comprehensive molecular characterization of muscle-invasive bladder. Cancer Cell. 2017;171:540-56. e25.

24. Tan TZ, Rouanne M, Tan KT, Huang RY, Thiery JP. Molecular subtypes of urothelial bladder cancer: results from a meta-cohort analysis of 2411 tumors. Eur Urol. 2019;75:423-32.

25. Rodriguez Pena MDC, Tregnago AC, Eich ML, Springer S, Wang $\mathrm{Y}$, Taheri D, et al. Spectrum of genetic mutations in de novo PUNLMP of the urinary bladder. Virchows Arch. 2017;471:761-7.

26. Nguyen D, Taheri D, Springer S, Cowan M, Guner G, Mendoza Rodriguez MA, et al. High prevalence of TERT promoter mutations in micropapillary urothelial carcinoma. Virchows Arch. 2016;469:427-34.

27. Kinde I, Bettegowda C, Wang Y, Wu J, Agrawal N, Shih Ie M, et al. Evaluation of DNA from the Papanicolaou test to detect ovarian and endometrial cancers. Sci Transl Med. 2013;5:167ra4.

28. Kinde I, Wu J, Papadopoulos N, Kinzler KW, Vogelstein B. Detection and quantification of rare mutations with massively parallel sequencing. Proc Natl Acad Sci USA. 2011;108:9530-5.

29. Kim PH, Cha EK, Sfakianos JP, Iyer G, Zabor EC, Scott SN, et al. Genomic predictors of survival in patients with high-grade urothelial carcinoma of the bladder. Eur Urol. 2015;67:198-201.

30. Hurst CD, Alder O, Platt FM, Droop A, Stead LF, Burns JE, et al. Genomic subtypes of non-invasive bladder cancer with distinct metabolic profile and female gender bias in KDM6A mutation frequency. Cancer Cell. 2017;32:701-15. e7.

31. Cowan M, Springer S, Nguyen D, Taheri D, Guner G, Rodriguez MA, et al. High prevalence of TERT promoter mutations in primary squamous cell carcinoma of the urinary bladder. Mod Pathol. 2016;29:511-5.

32. Cowan ML, Springer S, Nguyen D, Taheri D, Guner G, Mendoza Rodriguez MA, et al. Detection of TERT promoter mutations in primary adenocarcinoma of the urinary bladder. Hum Pathol. 2016;53:8-13.

33. Palsgrove DN, Taheri D, Springer SU, et al. Targeted sequencing of Plasmacytoid urothelial carcinoma reveals frequent TERT promoter mutations. Hum Pathol. 2018;85:1-9.

34. Pietzak EJ, Bagrodia A, Cha EK, Drill EN, Iyer G, Isharwal S, et al. Next-generation sequencing of nonmuscle invasive bladder cancer reveals potential biomarkers and rational therapeutic targets. Eur Urol. 2017;72:952-9. 